\title{
ON THE METHOD OF STATE INVARIANCE
}

\author{
Nguyen Canh Toan \& Do Van LaP
}

Institute of Information Technology

Summary. In this paper, we give a proof of proposition considered in privious one and study an optimal adaptive control.

\section{I. - PROOF OF PROPOSITION}

From (1.16) the times-derivative $\dot{E}$ can be written as

$$
\dot{E}=\dot{p}^{T} L p+p^{T} L \dot{p}+2 q^{T} M \dot{q}
$$

or, with $(1.15-1)$

$$
\dot{E}=p^{T}\left(A_{c}^{T} L+L A_{c}\right) p+q^{T} v b^{T} L p+p^{T} L b v^{T} q+2 q^{T} M \dot{q}
$$

Since

$$
p^{T}\left(L b v^{T} q\right)=\left(L b v^{T} q\right)^{T} p \quad \text { or } \quad p^{T} L b v^{T} q=q^{T} b v b^{T} L p
$$

then (18) becomes

$$
\dot{E}=p^{T}\left(A_{c}^{T} L+L A_{c}\right) p+2 q^{T}\left(v b^{T} L p+M \dot{q}\right) .
$$

For the condition

$$
\dot{q}=-Q^{-1} v b^{T} P p
$$

we have

$$
v b^{T} P p+Q \dot{q}=0
$$

Choosing the matrices $L=P, M=Q$, and according to (1.20), from (1.19) we obtain

$$
\dot{E}=p^{T}\left(A_{c}^{T} P+P A_{c}\right) p
$$

To show that $A_{c}$ is Hurwitz, let $n$-vector $p \neq 0$ and some $\lambda$ satisfy $A_{c} p=\lambda p$. Therefore [10]

$$
p^{T}\left(A_{c}^{T} P+P A_{c}\right) p=2 p^{T} P p \operatorname{Re} \lambda
$$


or, with (1.21)

$$
\dot{E}=2 p^{T} P p \operatorname{Re} \lambda
$$

Since $p^{T} P p>0$, according to (1.22) we have $\dot{E}<0$, as expected (1.17), if and only if the matrix $A_{c}$ is Hurwitz, i.e. if and only if $\operatorname{Re} \lambda<0$. In the case, there is always

$$
\dot{E}<0
$$

for $p \neq 0$, and

$$
\dot{E}=p^{T}\left(A_{c}^{T} P+P A_{c}\right) p .
$$

With (1.23), the extensive Ly apunov positive definite energy $E$ is a nondecreasing function of time which is bounded below and hence converges to some finite value $E_{00}$. Then according to (1.24) $\lim _{t \rightarrow \infty} \int_{0}^{t} \dot{E} d t=E_{00}-E(0)$ is a finite number and $\dot{E}$ is uniformly continuous since. $p$ and hence $\dot{E}$ is bounded. Hence by Barbalat's lemma [8] $\lim _{t \rightarrow \infty} \dot{E}=0$, or, with (1.24), we have $\lim _{t \rightarrow \infty} p^{T}\left(A_{c}^{T} P+P A_{c}\right) p=0$, therefore,

$$
\lim _{t \rightarrow \infty} p=0
$$

According to $(1.25)$ also by Barbalat's lemma $[8] \lim _{t \rightarrow \infty} \dot{p}=0$. With this result and with (1.25), from (1.15-1)

$$
\dot{p}=A_{c} p+b v^{T} q
$$

we can obtain

$$
\lim _{t \rightarrow \infty} q=0
$$

From given results (1.25) and (1.26) it is seen that the control plant

$$
\dot{p}=A_{c} p+B_{c}^{(\theta)} v w+z_{s}
$$

or the dynamic centralized process (1.26) in the condition

$$
\dot{q}=-Q^{-1} v b^{T} P p
$$

is asymptotically stable about zero in an extended state space $\{p, q\}$, if and only if matrix $\boldsymbol{A}_{c}$ is Hurwitz. Proposition has been proved. 
The condition for a stability $(1.29)$ is called the dynamic association equation of the process (1.26). After the dynamic association equation, the condition for the state inveriance of the plant can be formed as a state invariance of the plant with its centralized dynamic process (1.26) in the condition (1.29) is called the dynamic association equation of the process will be derived, if(1.27) occurs.

Actually, with (1.27) by Barbalat's lemma [8] we have

$$
\lim _{t \rightarrow \infty} \dot{q}=0
$$

According to this given result from the condition (1.29) we obtain (1.25) which implies the state invariance of the plant.

Thus, the control $w$ in the system (1.28) can be defined according to the conditions (1.27) we have (1.30) and (1.29). For the system (1.28) its control $w$ defined so that the conditions (1.29) and (1.30) are always satisfied and is called the adaptive algorithm. In this case the dynamic process (1.28) is said to be adaptive control process which will be able to guanrantee the state invariance of the given plant

$$
\dot{x}=A_{t} x+B_{1} u+z_{e}
$$

although there is the variance in its work condition.

Proposition 3. If there is asymptotic stability of the dynamic diametral process (1.26) and (1.29) about zero in the extended state space $\{p, q\}$ then the state invariance of the plant with its dynamic centralized system (1.28) will be derived. And on the contrary.

Actually, there is asymptotic stability of the process (1.26), (1.29) about zero in the state space $\{p, q\}$, i.e. there are (1.25) and (1.27), then, according to proposition 1, after the dynamic association equation, e (1.25) and (1.27) prove the state invariance of the given plant.

On the contrary, the state invariance of the plant with its dynamic centralized system (1.28) is derived, i.e. (1.25) occurs, then by Barbalat's lemma [8] we have $\lim _{t \rightarrow \infty} \dot{p}=0$. With thi result and with (1.33) from (1.32) it implies (1.28) or, with (1.12),

$$
\begin{aligned}
b v^{T} \Phi_{c}^{\left({ }^{\prime}\right)} \Delta \theta_{0} & =z_{*} \\
\Phi_{c}^{(0)}\left(w+\Delta \theta_{0}\right) & =q
\end{aligned}
$$

from (1.26) we can obtain (1.27). This given result and (1.25) prove that there is asymptotic satbility of the process (1.26) and (1.29) about zero in its state space $\{p, q\}$. 
Remark. If there is the state invariance of the plant, i.e. there is (1.25), then there will be asymptotic stability of the diametral process about zero in the state space $\{p, q\}$, i.e. there will be (1.27). Thus, in the case when

$$
\begin{aligned}
B & =(A, B), \quad . \\
v & =\left[\begin{array}{l}
x \\
u
\end{array}\right] \\
\Delta z & \equiv 0,
\end{aligned}
$$

if there is the state invariance of the plant then the vector - $w$ will be plant parameter error estimation vector

$$
-w=\Delta \theta, t \geq t_{c}
$$

with the great enough $t_{c}$.

\section{II. - THE STATE INVARIANCE ADAPTIVE CONTROL}

For the given plant each adaptive control system is constructed according to the concrete adaptive algorithm. And an Lena-Kumpati adaptive algorithm must be defined according to the main aim of the adaptive control process. It is the state invariance of the given plant or the condition for the its state invariance.

II.1. An optimal adaptive control.

The plant state invariance requirement (1.25) means that there exists such great $t_{c}$ enough that with $t \geq t_{c}$ there is always $p=0$. Therefore,

$$
V=p^{T} P p, t \geq t_{c}
$$

In this case the control $w$ of the process $(1.28)$ can be optimized and it usuably $[1],[2],[4],[5],[9]$ must minimize the Craxovski general work functional

$$
J=\frac{1}{2} \int_{t}^{t_{c}}\left(p^{T} P p+w^{T} K w\right) d t+w_{c}
$$

where $K$ is some positive definite $1 \times 1$-matrix

$$
W_{c}=w\left[p\left(t_{c}\right) w\left(t_{c}\right)\right]^{\bullet}
$$

According to the Belman dynamic program procedure the extensive kinetic quantity $\lambda$ of the process is defined by

$$
\lambda^{T}=\frac{\partial J}{\partial p} .
$$


Introduce (2.2) into (2.3) to obtain

$$
\lambda^{T}=\int_{t}^{t_{c}} p^{T} P p d t
$$

Then, with (2.1), (2.2) and (2.4) the Hamiltonian for this process

$$
H=\lambda^{T} \dot{p}+\frac{\partial J}{\partial p}
$$

has the shape

$$
H=\left(\int_{t}^{t_{c}} p^{T} P d t\right)\left(A_{c} p+B_{c}^{(\theta)} v w+z_{s}\right)-\frac{1}{2}\left(p^{T} P p+w^{T} K w\right)
$$

The optimal control $w$ is defined as the solution of the Hamilton equation

$$
\frac{\partial J}{\partial w}=0
$$

or, with (2.5)

$$
\left(\int_{t}^{t_{c}} p^{T} P d t\right) B_{c}^{(\theta)} v-W^{T} K=0
$$

therefore

$$
w=K^{-1}\left(B_{c}^{(\theta)} v\right)^{T} P \int_{t}^{t_{c}} p d t
$$

Since the Lena-Kumpati adaptive algorithm has to be defined on the basic of the condition for state invariance of the plant, therefore according to (2.6), with (1.29) and (1.30), the optimal adaptive algorithm can be established as following.

The control $w$ in the dynamic centralized process (1.28) of the plant (1.31) defined by $(1.26),(1.29)$ and

$$
w=K^{-1}\left(B_{c}^{(\theta)} v\right)^{T} P \int_{t}^{t_{c}} p d t
$$

is called the optimal adaptive algorithm if there exist some positive defined matrices $P, Q$ and $K$ such that the state invariance of the given plant is derived although there is the variance in its work condition.

In the case, when the extensive Craxovski general work functional

$$
J=\frac{1}{2} \int_{t}^{t_{c}}\left(p^{T} P p+w^{T} K w\right) d t+w_{c}
$$


is examined instead of the functional (2.3), with extensive kinetic quantity $\lambda$ defined by

$$
\lambda^{T}=\frac{\partial J}{\partial p}+\frac{\partial J}{\partial q} \frac{\partial q}{\partial p}
$$

and with

$$
\frac{\partial q}{\partial p}
$$

then the Lena-Kumpati optimal adaptive algorithm has the shape (1.26), (1.29) and

$$
\begin{aligned}
& w=K^{-1}\left(B_{c}^{(\theta)} v\right)^{T} \int_{t}^{t_{s}}\left(P p+R^{T} Q q\right) d t \\
& \dot{R}=-Q^{-1} v b^{T} P
\end{aligned}
$$

II.2. The extensive gradient adaptive algorithm

For given dynamic centralized process (1.28) of the plant (1.31)

$$
\dot{x}=A_{c} x+B_{c} u+z_{e}
$$

find the control $w$ which has to guarantee the state invariance of given plant.

Find it on the basic of the condition for the state invariance (1.27), (1.29) of the plant.

According to first condition (1.29) for the plant invariance (1.27), i.e. according to

$$
\lim _{t \rightarrow \infty} W=0
$$

where $W$ is extensive part of Lyapunov energy of extended diametral process, and

$$
W=q^{T} Q q
$$

we can search for such control $w$ which must minimize this energy $W$. The by the gradient principle $[4,5]$, the control $w$ can be defined by

$$
\frac{d w}{d t}=-\frac{K}{2}\left(\operatorname{grad}^{(w)} W\right)^{T}
$$

where $K$ is some diagonal positive definite $1 \times 1$-matrix.

Introduce (2.9) into (2.10) to obtain

$$
\dot{w}=-K\left(q^{T} Q \frac{\partial q}{\partial w}\right)^{T}
$$


or

$$
\dot{w}=-K R^{T} Q q
$$

noting

$$
\frac{\partial q}{\partial w}=R
$$

If the finction $q$ is cintinuous and differentiable then from second condition (1.29-2) for plant state invariance we can write

or, noting

$$
\begin{aligned}
\frac{\partial}{\partial w}\left(\frac{d}{d t} q\right) & =-Q^{-1} v b^{T} P \frac{\partial p}{\partial w} \\
\frac{d}{d t} \frac{\partial q}{\partial w} & =-Q^{-1} v b^{T} P \frac{\partial p}{\partial w}
\end{aligned}
$$

$$
\frac{\partial p}{\partial w}=S
$$

and with (2.12)

$$
\dot{R}=-Q^{-1} v b^{T} P S
$$

If the function $p$ is continuous and differentiable then from dynamic centralized equation (1.28) of the given plant, we can write

or, with (2.13),

$$
\begin{aligned}
\frac{\partial}{\partial w}\left(\frac{d}{d t} q\right) & =A_{c} \frac{\partial p}{\partial w}+B_{c}^{(\theta)} v \\
\frac{d}{d t} \frac{\partial q}{\partial w} & =A_{c} \frac{\partial p}{\partial w}+B_{c}^{(\theta)} v,
\end{aligned}
$$

$$
\dot{S}=A_{c} S+B_{c}^{(\theta)} v
$$

According to given results (2.11), (2.14) and (2.15), extensive gradient adaptive algorithm can be establish as (1.28) of the plant (1.31) defined by

$$
\begin{aligned}
& \dot{w}=-K R^{T} Q q, \\
& \dot{p}=A_{c} p+b v^{T} q \\
& \dot{q}=-Q^{-1} v b^{T} P p \\
& \dot{R}=-Q^{-1} v b^{T} P S \\
& \dot{S}=A_{c} S+B_{c}^{(\theta)} v
\end{aligned}
$$

is called the extensive gradient adaptive algorithm if there exist some positive definite matrices $P, Q$ and $K$ such that the state invariance of given plant is derived although there is the invariance in its condition. 
With (2.16), from (1.12-1) the formula for the design can be obtained as

$$
\begin{aligned}
B_{c} \varphi & =B_{c}^{(\theta)} v w \\
\dot{w} & =-K R^{T} Q q \\
\dot{p} & =A_{c} p+b v^{T} q \\
\dot{q} & =-Q^{-1} v b^{T} P p \\
\dot{R} & =-Q^{-1} v b^{T} P S \\
\dot{S} & =A_{c} S+B_{c}^{(\theta)} v,
\end{aligned}
$$

Thus treating centralized state equation of the plant and the condition for its state invariance the Lena-Kumpati adaptive algorithms have been established any model of given plant.

\section{III. - CONCLUSION}

In this paper the adaptive property of the control plant is characterized by new equation - the dynamic association equation to the dynamic centralized process of the plant. This equation is obtained analyzing conditions which have to be satisfied for the Lyapunov's stability theory to be successfully applied to adaptive control system. And conditions which have to be satisfied for the Lena-Kumpati adaptability idea are established.

Analyzing these conditions for the adaptability and treating dynamic centralized and association equations of the plant, the adaptive algorithms wich allow to construct the adaptive control systems which can guarantee the state invariance of the plant and therefore stabilized its dynamic adaptive process, that has been shown by proposition 3 , although there is the variance in its work condition, are established.

The simulating results correspond to theoretical claims.

\section{REFERENCES}

1. Dang Vu Giao, Automatic Control Theory: Fundamentals, Hanoi 1986.

2. Nguyen Huu Thung, Automation technique in Radio-communication, Hanoi 1987.

3. B.A. Bodner, Control system of the fly objects, Moscow 1988.

4. L.S. Design of the Radio-systems and Radio-equipments, Moscow, 1986.

5. A.A Kracovski, Analytic design of the adaptive control systems for the fly objects, Moscow 1973. 
6. A.W. Geofrey, On the local stability properties of adaptive parameter estimators with composite errors and split algorithm, IEEE transaction on automatic control v. 361991 .

7. J.M. Krause, Robust adaptive control: stability and asymptotic perfermance, IEEE trans. on automatic control, v. 371992.

8. S.N. Kumpti \&S.V. Lena, A comporison of Lyapunov and hyperstability approaches to adaptive control of continuous systems, IEEE trans. on automatic control v. 25 1980.

9. Z. Mariusz, Application of Lyapunov functional to studying stability of linear hyperbolic system, IEEE Trans. on automatic control v. 351990.

10. J.V. Robert, Design of reiable control systems, IEEC Trans. on automatic control v. 371992 .

11. C. Scherer, $H_{00}$ Control; by state-feedback and fast algorithms for the computation of $H_{00}$-nerms, IEEE Trans. on automatic control v. 351990.

12. M.A. Stephen, Towards a unified theory of parameter adaptive control, part II: certainly equivalents and implicit tuning, IEEE Trans. on automatic control v. 37 1992.

Institute of Information Technology

Institute of Technology

General defence industry office 